Instituto Internacional de Investigación y Desarrollo Tecnológico Educativo INDTEC, C.A.

DOI: https://doi.org/10.29394/Scientific.issn.2542-2987.2018.3.9.12.233-255

OAI-PMH: http://www.indteca.com/ojs/index.php/Revista Scientific/oai

Artículo Original / Original Article

\title{
Participación Ciudadana para una Educación Ambiental Sustentable
}

Autoras: Yuraima Margelis Matos De Rojas Universidad Nacional Experimental Simón Rodríguez, UNESR yuraimatos01@gmail.com

Trujillo, Venezuela

Eva Lidmila Pasek De Pinto Universidad Nacional Experimental Simón Rodríguez, UNESR mlinaricova@hotmail.com Trujillo, Venezuela Magda Lisbeth Peña Briceño Universidad Nacional Experimental "Rafael María Baralt", UNERMB bmagdaunica@hotmail.com

Trujillo, Venezuela Magda Violeta Briceño Universidad Nacional Experimental "Rafael María Baralt", UNERMB bmagda@hotmail.com.ar Trujillo, Venezuela

\section{Resumen}

El objetivo del estudio consistió en: Determinar las actividades de participación ciudadana que promueve el docente del L.B. "Sabana Libre" para el fortalecimiento de una educación ambiental sustentable. Teóricamente se fundamenta en la educación ambiental, el desarrollo sustentable y la participación ciudadana. Metodológicamente abordado desde la investigación descriptiva, realizado en el contexto de estudio. Se utilizó la técnica de la entrevista, a través de un cuestionario como instrumento para la recolección de la información, siendo validado por expertos. El instrumento se aplicó a cincuenta (50) docentes que laboran en el L.B. "Sabana Libre". Para el análisis se elaboró tablas con su respectiva interpretación estadística y descriptiva. Como resultados se obtuvo que $17 \%$ de docentes afirmó siempre realizar actividades pro-ambientales participativas y un $29 \%$ aseveró promover valores ambientales. Se concluye que poco se propicia la participación ciudadana como actividad del docente en la promoción de la educación ambiental sostenible y, en consecuencia, escasamente se promueve el desarrollo de una conciencia ambiental. Por lo que se sugiere la puesta en práctica de acciones que lleven a fortalecer la participación ciudadana hacia la valoración del ambiente. ambiente.

Palabras clave: participación comunitaria; ciudadanía; educación;

Fecha de Recepción: 05-03-2018

Fecha de Aceptación: 21-05-2018 


\section{Citizen Participation for a Sustainable Environmental Education}

Abstract

The objective of the study was to: determine the activities of citizen participation that promotes the teaching of the L.B. "Sabana Libre" for the strengthening of an environmental education sustainable. It is theoretically based on environmental education, sustainable development and citizen participation. Methodologically approached from the descriptive research, carried out in the context of study. We used the interview technique, through a questionnaire as an instrument for the collection of information, being validated by experts. The instrument was applied to fifty (50) teachers working in the L.B. "Sabana Libre". Tables with their respective statistical and descriptive interpretation were developed for the analysis. As results were obtained $17 \%$ of teachers said to always engage in participatory environmental activities and $29 \%$ said to promote environmental values. It is concluded that little conducive to citizen participation as an activity the teacher in the promotion of sustainable environmental education and, consequently, sparing the development of environmental awareness is promoted. So it is suggested the implementation of actions that lead to strengthening the participation to the valuation of the environment.

\section{Keywords: community participation; citizenship; education; environment.}




\section{Introducción}

La sustentabilidad constituye hoy día un reto que debe ser asumido en todos los contextos, de manera que garantice el desarrollo social, económico, científico de la humanidad. Ello exige promover prácticas de valores que estimulen patrones de consumo ecológicamente posibles para una mejor sociedad. En tal sentido, se busca satisfacer las necesidades humanas, incrementado el potencial productivo, a la vez asegurando oportunidades equilibradas que beneficien a toda la comunidad.

Dentro de los principales desafíos del desarrollo sustentable está el de propiciar una educación, formación y/o capacitación, que lleve a actuar conscientemente a todos los actores de la sociedad al resguardo, cuidado y mantenimiento del ambiente. Por lo tanto, se debe promover espacios educativos en el que se fomente el valor de la naturaleza cuyo norte sea alcanzar el desarrollo sustentable que demanda nuestro planeta.

Cabe referir que en la Constitución de la República Bolivariana de Venezuela (1999): el Artículo 15 preceptúa que "El Estado tiene la obligación de establecer una política integral preservando la diversidad y el ambiente". Así mismo, el Artículo 107:

La educación ambiental es obligatoria en los niveles y modalidades del sistema educativo, así como también en la educación ciudadana no formal. Es de obligatorio cumplimiento en las instituciones públicas y privadas, hasta el ciclo diversificado, la enseñanza de la lengua castellana, la historia y la geografía de Venezuela, así como los principios del ideario bolivariano.

En correspondencia con los estatutos legales, la Ley Orgánica de Educación (2009): en el Artículo 15, numeral 5, establece como uno de los fines de la educación "impulsar la formación de una conciencia ecológica para preservar la biodiversidad y la sociodiversidad, las condiciones ambientales y el aprovechamiento racional de los recursos naturales". Es decir, la educación 
es garante de la formación integral del ciudadano y ciudadana y en especial hacia la acción ambiental. Es por ello, que la educación juega un rol importantísimo en la formación de valores ambientales, garantizando de esta manera la sustentabilidad y un mejor porvenir.

En este orden de ideas y dado que, la educación ambiental debe ser impartida en los diferentes niveles educativos, los docentes han de contribuir con la formación integral del estudiante. Dicha formación incluye preparar el talento humano necesario, tanto en las diferentes disciplinas, las cuales se traducen en mayores y mejores conocimientos para alcanzar las demandas de un país en proceso de desarrollo, como formar los ciudadanos críticos, con conciencia ecológica, que valoren el ambiente y actúen para preservar y resguardar el planeta, nuestra "Tierra-Patria" (Morin, 2000).

Ante la relevancia de la educación ambiental en los actuales momentos, Pasek (2006a): expresa que los docentes deben poseer un nivel crítico de conciencia ambiental y Matos (2013): indica que el docente de aula tiene que asumir su rol orientador y social para un accionar más eficiente, hacia una educación más humanista y ambientalista, frente a los cambios que exige la actual sociedad. Desde esta perspectiva, el docente como gerente de aula debe promover acciones que contribuyan al fortalecimiento del proceso de aprendizaje en las diferentes áreas del saber, en la medida que integre los conocimientos con las experiencias para educación ambiental sustentable.

Así mismo, el docente debe, como señalan Jara y Parada (2011a): promover actividades ecológicas que lleven a propiciar la participación ciudadana para una educación ambiental sustentable, indicando entre ellas: las pro-ambientales y la promoción de valores ambientales. En este sentido se espera que el docente promueva actividades que le permitan involucrar a los actores sociales en las diversas actividades hacia el fomento de una educación ambiental y sustentable.

A pesar de la preocupación del Estado y el Sistema Educativo 
venezolano por una educación ambiental humanista y sustentable, se viene observando en estudios como el de Navea (2018a): en su estudio concluye que se requiere considerar acciones ambientalistas que lleven a construir conocimientos, donde se apropien de los mismos, para que se adquiera el compromiso de la comunidad hacia la valoración ambiental como elemento interviniente para lograr la calidad de vida. Por su parte, Mendoza (2018), refiere en su estudio que son pocas las actividades que propician para el embellecimiento de la comunidad, porque hay escasez de formación en la comunidad en general.

Situación que llevó a plantear como objetivo del estudio, determinar las actividades de participación ciudadana que promueve el docente del L.B. "Sabana Libre", para el fortalecimiento de una educación ambiental sustentable. Investigación abordada desde el paradigma positivista a través del método descriptivo.

\section{Bases Teóricas}

\subsection{Educación ambiental}

La educación ambiental viene a constituir una estrategia educativa que lleva a ambientalizar desde y con los centros educativos y la comunidad o contexto, para la obtención de un conocimiento, una toma de consciencia que permita establecer estructuras participativas para la resolución de problemas ambientales producto del mal accionar del ser humano. Se trata de establecer cambios que lleven a pensar ecológicamente en bienestar de nuestro ambiente. Pasek (2005a), señala que el Ministerio del Poder Popular para el Ambiente (MPPA), indica que:

La Educación Ambiental se define en Venezuela como el proceso que posibilita la formación de un hombre capaz de comprender la complejidad producida en el ambiente por la interacción de sus componentes naturales y socioculturales, a 
la vez que le permite ser crítico, emitir juicios de valor y adoptar normas de comportamiento cónsonas con estos juicios; además, se entiende como la realización de un conjunto de actividades integradas en un proceso sistemático y permanente, desarrolladas a través de múltiples medios, dirigidas a promover cambios de comportamiento en todos los sectores de la población, que evidencian la adopción de nuevos valores orientados hacia la conservación, defensa y mejoramiento del ambiente cuya finalidad última sea, mejorar en forma constante las condiciones de vida de las generaciones actuales y futuras. (pág. 36).

Desde lo señalado por las autoras, se trata de crear una cultura ambientalista que contribuya a valorar y conservar el espacio en el que se convive. En este sentido, se trata de ofrecer oportunidades de participación corresponsable donde los actores de la escuela y comunidad se conviertan en los propios autores del resguardo, cuidado y desarrollo de su ambiente. Pues como señalan Dieleman y Juárez (2008): se trata de asumir medidas adecuadas que lleven a la implicación decidida de todos los actores de la sociedad para fortalecer una conciencia ambientalista, que forme hacia una nueva forma de relación del hombre-mujer hacia el resto de la naturaleza.

En este sentido, se requiere de una constante formación y educación, que como señala Navea (2018b): "debe partir desde la normativa de la moral que exige la responsabilidad por parte de todos los ciudadanos, en cuanto al cuidado del entorno natural, donde se procure el bienestar entre la sociedad y la naturaleza" (pág. 138). El interés está puesto en la conciencia, que lleve a la puesta en práctica de los conocimientos vayan adquiriendo en el proceso de formación para un desarrollo sustentable ambiental efectivo.

\subsection{Desarrollo sustentable}

El desarrollo sustentable, para Gabaldón (2006): debe llevar a generar 
prosperidad económica, sustentada en unos principios éticos, que debe

ponerse en práctica en todos los niveles educativos desde una perspectiva holística, considerando el presente y el futuro, en beneficio de la sociedad a nivel nacional e internacional. Para la Ley Orgánica del Ambiente (2006), en su Artículo 3:

se entiende el desarrollo sustentable como un proceso de cambio continuo y equitativo para lograr el máximo bienestar social, mediante el cual se procura el desarrollo integral, con fundamento en medidas apropiadas para la conservación de los recursos naturales y el equilibrio ecológico, satisfaciendo las necesidades de las generaciones presentes sin comprometer las generaciones futuras.

En Venezuela, desde los diferentes estamentos legales se viene promoviendo el desarrollo de un mejor ambiente, que sea sustentable para el bienestar de la sociedad, donde se hagan participes activos todos los actores que confluyen en la escuela y comunidad, resaltando el grado compromiso que tiene el estado para que se lleve a efecto de manera satisfactoria. Por tales razones, se debe formar al estudiante como a la comunidad, desde una visión integradora, que lo lleve a reflexionar sobre las repercusiones ambientales de sus actos; incorporando a los actores socioeducativos para que actúen en beneficio de la institución, comunidad y Estado hacia el rescate y preservación del ambiente.

En consecuencia, el docente debe promover la participación activa que coadyuve a una formación ambientalista para la preservación de la vida y de las generaciones futuras. Se trata en sí de generar estrategias que lleve a participar con sentido de responsabilidad, corresponsabilidad y solidaridad hacia la puesta en práctica del trabajo en equipo desde la triada familiaescuela y comunidad por una mejor sociedad. 


\subsection{Participación ciudadana}

La participación para Pasek, Ávila y Matos (2015): consiste en la intervención de las partes interesadas para realizar en colectivo acciones que lleven al disfrute de los resultados. Desde el accionar en conjunto se puede generar relaciones armoniosas que les permita la búsqueda de soluciones a hechos concretos. Por lo tanto, se puede decir que La participación es un derecho y un deber de los ciudadanos en beneficio de sí mismo como del contexto donde se desenvuelve.

En cuanto a la participación ciudadana, de acuerdo con Jara y Parada (2011b): es un proceso formativo que lleva a un accionar de forma correcta ante el mundo. Por su parte, Peña (2018a: 204): señala que es "un proceso mediante el cual los habitantes de una nación tienen el derecho de intervenir individual y colectivamente en las diversas acciones comunes que les afecte". Como se puede apreciar, se trata los actores de la escuela y comunidad trabajen mancomunadamente para el logro de las metas y, más aún, para conservar el ambiente en todas sus dimensiones: contexto, espacio, relaciones. Consiste en hacer participar a la ciudadanía en las diferentes actividades que lleva a cabo la escuela para que responsablemente actúen en beneficio de su escuela y comunidad.

\subsection{Actividades ecológicas para la participación ciudadana}

Las actividades, para Gelvis (2017): están referidas al conjunto de fenómenos de la vida activa que tienden hacia una finalidad, vienen a constituir la sustancia de la conciencia humana. Por lo que la autora señala que la actividad social es considerada como "un sistema de dinamización que permite la participación constante de todos los actores socioeducativos para desarrollar las capacidades creativas del individuo donde se busque el crecimiento participativo en los procesos social de una comunidad" (pág. 58). Es decir, la participación activa que deben generar los docentes desde los 
espacios educativos para fortalecer una educación ambiental sustentable.

En este sentido, es necesario promover actividades ecológicas donde se involucre a los actores sociales y educativos para que se integren al trabajo por el rescate del ambiente. Al respecto, Jara y Parada (2011c) y Tovar (2012a): sugieren como actividades ecológicas las pro-ambientales y las de promoción de valores ambientales para contribuir con una formación ecológica que lleve a disminuir la desigualdad social, ofrezca apoyo comunitario y promueva un ambiente sano.

En relación con las actividades pro-ambientales, son todas aquellas que se pueden realizar con las comunidades para el beneficio del entorno escolar y comunitario. Entre ellas, se pueden señalar: el reciclaje de residuos sólidos y su ubicación en envases identificados. En la misma línea, se pueden incluir otros estudios que atañen a la disminución en la producción de basura doméstica, como el de Osorio (2018a): así como trabajos que señalan condiciones para propiciar que las personas depositen sus desechos en los lugares destinados para su recogida (Pasek, 2005b). Son diversos los trabajos que se han realizado en escenarios públicos como parques, centros deportivos, cafeterías, entre otros; haciendo énfasis en la necesidad de:

- Promover acciones con la comunidad para el rescate de las áreas verdes.

- Establecer comisiones para que ayuden a conservar el ambiente.

- Organizar a la comunidad y estudiantes para separar objetos y reciclar.

- Propiciar la colocación de los materiales en recipientes adecuados.

En cuanto a la promoción de valores y con base al educar en valores, el docente debe promover valores ambientalistas. Se entiende que la educación está "omnipresente en la existencia cotidiana de los seres humanos" y "la presencia de algún modo de educación es constante en la vida 
de los individuos" (Ibáñez, 2004a). El valor está presente en el acontecer diario de todo ser humano.

Es por lo que, para educar en valores se debe prever el contexto, así como considerar lo afectivo y cognitivo del ser (Ibáñez, 2004b). Por lo tanto, la promoción de valores debe fomentar actitudes y comportamientos que favorezcan la consolidación de una conciencia ética y estética y formar ciudadanos con conciencia local y planetaria quienes, al interactuar con las distintas formas de vida, respete sus ciclos vitales (Pasek, 2005c).

La educación para Jara y Parada (2011d): debe estar orientada al fortalecimiento de ciudadanos críticos, responsables y comprometidos consigo mismo, con el otro y con la sociedad donde se desenvuelve. Por eso, los docentes deben propiciar actividades tales como:

- Promover valores ambientales hacia el resguardo y rescate del ambiente.

- Realizar reuniones con padres y representantes para ahorrar la energía.

- Propiciar conversatorios sobre la conservación del agua.

- Incorporar los objetivos institucionales para establecer corresponsabilidad con las actividades ecológicas.

En síntesis, se trata de promover una educación que incorpore a los actores educativos en actividades pro-ambientales y promoción de valores, que supere un discurso pedagógico formal y burocrático. Se desea formar ciudadanos capaces de cuestionar y actuar en beneficio de su ambiente.

\section{Metodología}

El estudio se insertó en el paradigma cuantitativo asumiendo una investigación de tipo descriptivo que, de acuerdo con Hernández, Fernández 
y Baptista (2010:80): "busca especificar propiedades, características y rasgos importantes de cualquier fenómeno que se analice. Describe tendencias de un grupo o población". El diseño fue de campo no experimental, el cual para Sabino (2007:81): "permite cerciorarse al investigador de las verdaderas condiciones en que se han conseguido los datos, posibilitando su revisión o modificación en el caso de que surjan dudas respecto a su calidad". En tal caso, la información se recolectó en el contexto de estudio L.B. "Sabana Libre".

Se aplicó como técnica la encuesta a través de un cuestionario, que para Hurtado (2012): viene a constituir un instrumento donde se agrupan una serie de preguntas inherentes a la situación de estudio o temática particular de la que desea obtener información. Para efecto del mismo, se elaboró un cuestionario tipo escala, contentivo de 8 (ocho) ítems con cinco alternativas de respuesta: (5) Siempre, (4) Casi siempre, (3) Algunas veces, (2) Rara vez y (1) Nunca.

El instrumento fue validado por expertos en la materia y en investigación, quienes hicieron sugerencias acordes con las variables del estudio. Las mismas se realizaron y se procedió a su validación respectiva. Para obtener la confiabilidad del instrumento se utilizó el método alfa de Cronbach, el cual consiste en aplicar el instrumento una sola vez a un grupo piloto con características similares a la población de estudio. Utilizando el programa estadístico Statistical Package for the Social Sciences (SPSS) versión 15.0, se obtuvo un coeficiente de $0,92 \%$ que, por estar cerca de 1 , se consideró altamente confiable para su aplicación.

\section{Análisis e interpretación de resultados}

El análisis descriptivo porcentual se presenta organizado en dos tablas, una para las actividades pro-ambientales y la otra para la promoción de los valores ambientales. El cuidado ambiental y la participación están preceptuados en distintos documentos legales, por eso, se asume que todos 
los docentes deberían cumplir con ello y realizar siempre actividades proambientales y de promoción de valores ambientales.

Tabla 1. Actividades Pro-ambientales

\begin{tabular}{|c|c|c|c|c|c|c|c|c|c|c|}
\hline \multirow{2}{*}{ ITEMS } & \multicolumn{2}{|c|}{ SIEMPRE } & \multicolumn{2}{c|}{ CASI } \\
SIEMPRE & \multicolumn{2}{c|}{$\begin{array}{c}\text { ALGUNAS } \\
\text { VECES }\end{array}$} & \multicolumn{2}{c|}{$\begin{array}{c}\text { RARA } \\
\text { VEZ }\end{array}$} & \multicolumn{2}{c|}{ NUNCA } \\
\cline { 2 - 11 } & F & $\%$ & F & $\%$ & F & $\%$ & F & $\%$ & F & $\%$ \\
\hline $\begin{array}{l}\text { 1. Promueve acciones con la } \\
\text { comunidad para el rescate de } \\
\text { las áreas verdes en plazas, } \\
\text { parques, otros. }\end{array}$ & 11 & 22 & 9 & 18 & 8 & 16 & 15 & 30 & 7 & 14 \\
$\begin{array}{l}\text { 2. Establece grupos de } \\
\text { trabajo para la conservación } \\
\text { ambiental. }\end{array}$ & 8 & 16 & 14 & 28 & 18 & 36 & 7 & 14 & 3 & 6 \\
$\begin{array}{l}\text { 3. Organiza a la comunidad y } \\
\text { estudiantes para separar } \\
\text { objetos o reciclar. } \\
\text { 4. Propicia la colocación de } \\
\text { los materiales en recipientes } \\
\text { adecuados. }\end{array}$ & 9 & 18 & 9 & 18 & 7 & 14 & 10 & 20 & 15 & 30 \\
$\quad$ PROMEDIO & 9 & 17 & 11 & 22 & 11 & 22 & 11 & 22 & 9 & 17 \\
\hline
\end{tabular}

Fuente: Resultados del instrumento (2017).

En cuanto a la Participación Ciudadana en lo que concierne a las actividades pro-ambientales, se puede decir que el $30 \%$ rara vez realiza acciones con la comunidad para el rescate de las áreas verdes en plazas, parques, otros, el $22 \%$ que siempre, el $18 \%$ casi siempre, un $16 \%$ algunas veces y el $14 \%$ que nunca. En el segundo ítem, el $36 \%$ señaló que algunas veces establecen grupos de trabajo para la conservación ambiental, el 28\% que casi siempre, un $16 \%$ que siempre, un $14 \%$ casi nunca y un $6 \%$ que nunca.

Para el tercer ítem, sobre si organizan a la comunidad y estudiantes para separar objetos o reciclar, el $30 \%$ de los encuestados señalaron que nunca lo realiza, un $20 \%$ rara vez, un $18 \%$ siempre y otro $18 \%$ casi siempre, y un $14 \%$ algunas veces. En lo que corresponde al propiciar la colocación de los materiales en recipientes adecuados, el $26 \%$ indicó que rara vez lo hacen, el 
$22 \%$ que nunca, otro $22 \%$ que casi siempre, un $18 \%$ algunas veces y un $10 \%$ que siempre.

Se puede apreciar que las actividades pro-ambientales, sólo las aplica siempre un $17 \%$ de los docentes encuestados. Por lo que cumplen con los preceptos legales y según Jara y Parada (2011e) y Tovar (2012b): se benefician el entorno escolar y comunitario.

Por otra parte, se tiene a la mayoría ( $83 \%$ en promedio) que no promueven un ambiente saludable y sustentable, ni el desarrollo de una conciencia ambientalista, puesto que no involucran a todos los ciudadanos de la localidad para incorporarse al trabajo ecológico y así preservar el ambiente (Osorio, 2018b). Luego, poco se está logrando una educación ambiental sustentable.

Tabla 2. Promoción de valores ambientales

\begin{tabular}{|c|c|c|c|c|c|c|c|c|c|c|}
\hline \multirow[t]{2}{*}{ ITEMS } & \multicolumn{2}{|c|}{ SIEMPRE } & \multicolumn{2}{|c|}{$\begin{array}{c}\text { CASI } \\
\text { SIEMPRE }\end{array}$} & \multicolumn{2}{|c|}{$\begin{array}{l}\text { ALGUNAS } \\
\text { VECES }\end{array}$} & \multicolumn{2}{|c|}{$\begin{array}{l}\text { RARA } \\
\text { VEZ }\end{array}$} & \multicolumn{2}{|c|}{ NUNCA } \\
\hline & $\mathbf{F}$ & $\%$ & $\mathbf{F}$ & $\%$ & $F$ & $\%$ & $\mathbf{F}$ & $\%$ & $\mathbf{F}$ & $\%$ \\
\hline $\begin{array}{l}\text { 5. Promueve la valoración del } \\
\text { ambiente con los actores de la } \\
\text { escuela y comunidad. }\end{array}$ & 18 & 36 & 16 & 32 & 9 & 18 & 3 & 6 & 4 & 8 \\
\hline $\begin{array}{l}\text { 6. Fortalece valores ambientales } \\
\text { como el ahorro de la energía. }\end{array}$ & 10 & 20 & 8 & 16 & 13 & 26 & 6 & 12 & 13 & 26 \\
\hline $\begin{array}{l}\text { 7. Propicia conversatorio sobre la } \\
\text { conservación del agua. }\end{array}$ & 14 & 28 & 19 & 38 & 9 & 18 & 4 & 8 & 4 & 8 \\
\hline $\begin{array}{l}\text { 8. Establece la corresponsabilidad } \\
\text { en las actividades ecológicas. }\end{array}$ & 16 & 32 & 8 & 16 & 19 & 38 & 3 & 6 & 4 & 8 \\
\hline PROMEDIO & 15 & 29 & 13 & 26 & 13 & 26 & 4 & 8 & 6 & 12 \\
\hline
\end{tabular}

Fuente: Resultados del instrumento (2017).

En lo que concierne a la promoción de valores ambientales, en el ítem 5 , dirigido a la valoración del ambiente con los actores de la escuela y comunidad, se aprecia que un $36 \%$ de los encuestados señalaron: siempre lo realizan, un $32 \%$ refirió que casi siempre, un $18 \%$ algunas veces, el $8 \%$ que 
nunca y el $6 \%$ casi nunca. En cuanto al ítem 6 relativo al fortalecimiento de valores como el ahorro de la energía, el $26 \%$ de los encuestados señalaron que nunca, otro $26 \%$, que algunas veces, el $20 \%$ que siempre, un $16 \%$ casi siempre y $12 \%$ rara vez.

En cuanto al ítem 7, sobre si propician conversatorios sobre la conservación del agua, el $38 \%$ de los entrevistados señalaron que casi siempre, un $28 \%$ que siempre, un $18 \%$ que algunas veces y un $8 \%$ que casi nunca y otro $8 \%$ que nunca. Para el ítem 8 , donde se establece la corresponsabilidad en las actividades ecológicas, el $38 \%$ señaló que algunas veces lo hacen, un $32 \%$ que siempre, un $16 \%$ que casi siempre, un $8 \%$ que nunca y un $6 \%$ que casi nunca.

De los resultados se puede inferir que, en promedio sólo $29 \%$ de los docentes encuestados promueven valores para una educación ambiental sostenible pues realizan las actividades pertinentes siempre. Esto se corresponde con lo señalado por Ibáñez (2004c) y (Jara y Parada, 2011f): al referir que el docente debe educar en valores, orientándolos a realizar las elecciones más acertadas en beneficio de sí mismo, de la sociedad y del ambiente.

Por otro lado, existe un $79 \%$ de docentes que acepta no realizar siempre las actividades de promoción de valores ambientales. Este resultado permite deducir que, no se está formando un ciudadano con conciencia ambientalista, ni se potencia tal conciencia en la comunidad. En promedio general se tiene, entonces, que sólo $23 \%$ de los docentes aparentemente propician la participación ciudadana para la promoción de una educación ambiental sustentable. Situación que contradice a Peña (2018b), cuando refiere que la escuela y comunidad deben promover la participación ciudadana, donde se integren voluntariamente a las actividades en la medida que se consoliden equipos de trabajo, para desarrollar las capacidades individuales y colectivas con el fin de lograr una meta. 
Igualmente contradice a Garza y Patiño (2004): quienes indican que la participación ciudadana desde la escuela debe involucrar tanto a los estudiantes como a representantes y comunidad en el desarrollo de actividades sociales en beneficio del entorno o comunidad donde viven. Se trata de fortalecer la participación activa de los actores socioeducativos hacia una educación ambiental sustentable y una conciencia ecológica en beneficio de la humanidad.

\section{Conclusiones}

En Venezuela, tanto la participación ciudadana como la educación ambiental están preceptuadas en diferentes documentos legales. Quiere decir, que desde las instituciones educativas se debe promover ambas y el docente, en tanto gerente del aula, es el responsable de realizar actividades para ello. No obstante, al determinar las actividades de participación ciudadana que promueven al docente del L.B. "Sabana Libre" para el fortalecimiento de una educación ambiental sustentable, se encontró que, en promedio, solo 17\% de los docentes realiza actividades pro-ambientales y el $29 \%$ promueve valores ambientales implicando la triada escuela-familia-comunidad. Tales resultados permiten concluir que:

1.- La mayoría de los docentes no están formando un ciudadano crítico y reflexivo, capaz de pensar sobre su propia responsabilidad en el deterioro del ambiente.

2.- Aunque la educación ambiental es obligatoria, en su mayoría, los docentes no están educando en valores ambientales ni realizan actividades pro-ambientales. Por lo tanto, ni los estudiantes ni la comunidad aprenden a respetar, cuidar y preservar el entorno y el ambiente en general.

3.- Consecuentemente, no se favorece el desarrollo de una conciencia ambiental y planetaria que lleve a los estudiantes y a la comunidad a preservar y asumir compromisos ambientales. En pocas palabras, no estamos 
enseñando y aprendiendo a cuidar nuestra "Tierra-Patria".

4.- Finalmente, vistos los resultados, aparentemente no estamos en dirección hacia un desarrollo sustentable, pues éste implica unos principios éticos que se deben practicar en colectivo y orientar las actividades del ser humano en su interacción con el entorno, tomando en consideración el presente y el futuro. Pero no estamos formando hoy, en el presente, la generación futura en dichos principios ni de manera participativa.

Debido a ello y para cerrar, es importante sugerir algunas actividades ecológicas, cuya práctica vincule de manera participativa y ética a la escuela, por medio de docentes y estudiantes, con la familia y la comunidad. Retomamos las actividades pro-ambientales y de promoción de valores ya mencionadas como el rescate de las áreas verdes, comisiones de trabajo para conservar el ambiente, el reciclaje de los objetos, la colocación de los materiales en recipientes adecuados; sensibilizar para evitar la contaminación; tomar conciencia sobre el valor de la conservación del agua y del ahorro de la energía de sus hogares. A éstas es posible agregar:

1. Elaborar un plan de cuidado y mantenimiento ambiental que promueva la participación entre el colectivo de la escuela y comunidad de manera que lo puedan llevar a cabo de manera corresponsable.

2. Generar espacios de discusión en el aula y la comunidad sobre los problemas ambientales que existen actualmente en la comunidad y en los diferentes espacios del planeta. Esto favorece la reflexión y la toma de conciencia al respecto.

3. Revisar y avisar sobre botes de agua en las instalaciones de la escuela.

4. Conformar un club o una brigada de conservacionismo ambiental.

5. Organizar un club de excursionismo ecológico.

6. Organizar con los estudiantes comisiones de vigilancia ambiental escolar que revisen pasillos, botes de agua, luces encendidas, jardines. 
7. Organizar con la comunidad un proyecto de reciclaje. Dependiendo del tipo de desecho podría, inclusive, generar ingresos para la escuela.

8. Planificar en cada lapso al menos un proyecto de aprendizaje centrado en el ambiente y su cuidado.

Son sólo algunas posibles, su número y su práctica dependerán de las necesidades e intereses de los actores socioeducativos involucrados. Para cerrar, es pertinente resaltar que se trata de tomar el ambiente como parte y soporte de nuestras vidas; lo que implica estudiarlo para conocerlo y optar por su valor, transformándolo para humanizarlo sin devastarlo (Pasek, 2006b). Es decir, plantear la protección del entorno para un mejor futuro.

\section{Referencias}

Constitución de la República Bolivariana de Venezuela (1999). Gaceta Oficial N. ${ }^{\circ}$ 36.860, del 30 de diciembre. Caracas, Venezuela: Asamblea Nacional Constituyente.

Dieleman, H. \& Juárez, N. (2008). Educación ambiental para la sustentabilidad. Plan de estudio. México: Dirección General de Educación Superior para Profesionales de la Educación.

Gabaldón, A. (2006). Desarrollo sustentable, la salida de América Latina. Venezuela: Grijalbo.

Garza, J., \& Patiño, S. (2004). Educación en Valores. México: Trillas. Gelvis, A. (2017). Actividades Recreativas para Fortalecer el Rendimiento Escolar de los Estudiantes. Revista Scientific, 2(4), 46-63. Recuperado de:

https://doi.org/10.29394/scientific.issn.2542-2987.2017.2.4.3.46-63

Hernández, R., Fernández, C., \& Baptista, M. (2010). Metodología de la investigación. (5ta ed.). México: McGraw-Hill.

Hurtado, J. (2012). Paradigmas y Métodos de Investigación en Tiempos de 
Cambio. Caracas, Venezuela: SYPAL.

Ibáñez, G., T. (2004a,b,c). Introducción a la Psicología Social. España: UOC.

Jara, N., \& Parada, R. (2011a,b,c,d,e,f). Rol del gerente de aula en la promoción de actividades ecológicas en la educación primaria. Daena: International Journal of Good Conscience, 6(2), 34-53, México: Spenta University Mexico, ISSN: 1870-557X. Recuperado de: http://www.globethics.net/gel/4888451

Ley Orgánica de Educación (2009). Gaceta Oficial N. 5.929, extraordinaria del 15 de agosto. Caracas, Venezuela: La Asamblea Nacional de la República Bolivariana de Venezuela. Recuperado de:

https://www.urbe.edu/portal-biblioteca/descargas/Ley-Organica\%20deEducacion.pdf

Ley Orgánica del Ambiente (2006). Gaceta Oficial de la República Bolivariana de Venezuela, año CXXXIV, mes III, N..$^{\circ}$ 5.833, extraordinaria del 22 de diciembre. Recuperado de:

http://www.oas.org/juridico/PDFs/mesicic4 ven lo ambi.pdf

Matos, Y. (2013). Actividades de participación para una recreación sana sustentable. Sustentabilidad al día, (2), 1-13. Trujillo, Venezuela: Universidad Valle del Momboy, Depósito Legal pp: 201202TR4202.

Mendoza, Y. (2018). Fortalecimiento de la educación ambiental a través de la siembra de plantas ornamentales. Revista Scientific, 3(7), 309328. Recuperado de: https://doi.org/10.29394/Scientific.issn.25422987.2018.3.7.16.309-328

Morin, E. (2000). Los siete saberes necesarios a la educación del futuro. Venezuela: Coedición FACES-UCV y IESALC/UNESCO.

Navea, J. (2018a,b). Acciones ambientalistas para la conservación de las cuencas hidrográficas. Revista Scientific, 2(6), 134-153. Recuperado de: https://doi.org/10.29394/scientific.issn.2542-2987.2017.2.6.7.134- 
Osorio, E. (2018a,b). Acciones ambientalistas en la recolección de la basura doméstica. Revista Scientific, 2(6), 113-133. Recuperado de: https://doi.org/10.29394/scientific.issn.2542-2987.2017.2.6.6.113-133

Pasek, E (2005a,b,c). Hacia una conciencia ambiental. Revista Educere, 8(24),34-40, ISSN: 1316-4910, Mérida, Venezuela: Universidad de Los Andes. Venezuela: Saber ULA. Recuperado de:

http://www.saber.ula.ve/handle/123456789/19834

Pasek, E. (2006a,b). El docente y su nivel de conciencia ambiental. Revista de Artes y Humanidades UNICA, 7(15), 79-94, ISSN: 1317-102X. Recuperado de: http://www.redalyc.org/articulo.oa?id=170118694005

Pasek, E., Ávila, N., \& Matos, Y. (2015). Concepciones sobre participación social que poseen los actores educativos y sus implicaciones. Revista Paradigma, 36(2), 99-121. Recuperado de:

http://revistas.upel.edu.ve/index.php/paradigma/article/view/3017/1429

Peña, S. (2018a,b). La participación ciudadana como vía para abordar la acción transformadora de la escuela primaria. Revista Scientific, 3(8), 197-217. Recuperado de: https://doi.org/10.29394/Scientific.issn.2542$\underline{2987.2018 .3 .8 .10 .197-217}$

Sabino, C. (2007). El proceso de Investigación. Caracas, Venezuela: Panapo.

Tovar, J. (2012a,b). Hacia una educación ambiental ciudadana contextualizada: consideraciones teóricas y metodológicas desde el trabajo por proyectos. Revista Iberoamericana de Educación, 58(2), 1-11, ISSN-e: 1681-5653, ISSN: 1022-6508. Recuperado de: https://dialnet.unirioja.es/servlet/articulo?codigo $=5894707$ 


\section{Yuraima Margelis Matos de Rojas}

e-mail: yuraimatos01@gmail.com

Nacida en Venezuela. Licenciada en Educación Integral,

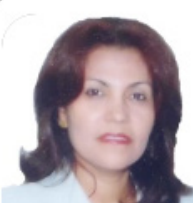

Mención Matemáticas, Magister en Ciencias de la Educación. Mención Investigación Docencia. Magister en Tecnología y Diseño educativo, Magister en Educación Robinsoniana, Dra. En Ciencias de la Educación. Docente investigador en la Universidad Nacional Experimental Simón Rodríguez, (UNESR) Núcleo Valera, Edo. Trujillo (Jubilada). Coordinadora de la Línea de investigación: Investigadores en acción Social (IAS), Facilitadora en cursos de metodología, Seminario de investigación, Valores, Servicio Comunitario, Proyecto de Investigación, administración de la educación Básica, Ética y valores en la investigación, gerencia educativa, entre otros cursos, en las Universidades: Simón Rodríguez, Valle del Momboy, Rafael María Baralt (Especialidad, Maestría y Doctorado). Jurado evaluador y tutora de Trabajo Especial de Grado y Tesis, Evaluadora de trabajos de ascenso, artículos arbitrados en Revistas nacionales e internacionales. Actualmente miembro del PEII, NIVEL B. 


\section{Eva Lidmila Pasek De Pinto}

e-mail: mlinaricova@hotmail.com

Nacida en Caracas, Venezuela. Licenciada en Educación

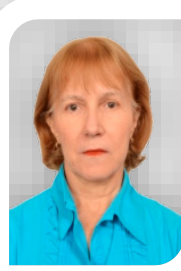

Mención Ciencias Biológicas (UCAB), Magister en Planificación y Administración de la Educación Superior URU), Magister en Tecnología y Diseño Educativo (UNESR), Especialista en Metodología de la Investigación (URU), Doctora en Ciencias de la Educación (UBA); Estudios Postdoctorales en Ciencias de la Educación (UNESR). Docente jubilada por la Universidad Nacional Experimental Simón Rodríguez. Responsable de proyectos sobre construcción del conocimiento y ciencias naturales; ambiente y evaluación. Miembro activo de la Línea de Investigación "Investigadores en Acción Social" (IAS). Tiene publicado un libro como autora-editora y numerosos artículos en revistas nacionales e internacionales. Docente investigadora del Programa de Estímulo a la Innovación e Investigación en el Nivel C. 


\section{Magda Lisbeth Peña Briceño}

e-mail: bmagdaunica@hotmail.com

Nacida en Trujillo, Venezuela. Licenciada en Educación Integral, Licenciada en Educación

Preescolar, Magister en Administración de la Educación Básica, Estudiante del Doctorado en Educación de la Universidad Nacional Experimental "Rafael María Baralt". Laboro actualmente en U.E. 
Magda Violeta Briceño

e-mail: bmagda@hotmail.com.ar

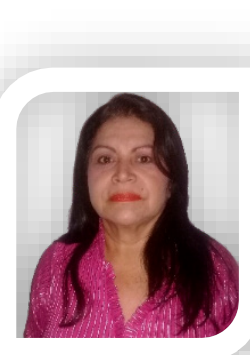

Nacida en Trujillo, Venezuela. Licenciada Mención

Biología, Licenciada en Educación Preescolar,

Magister en Administración de la Educación Básica,

Estudiante del Doctorado en Educación de la Universidad Nacional Experimental "Rafael María Baralt". Laboro actualmente como directora en la U.E. José Gregorio Hernández. 